\title{
Musculoskeletal manifestations of Lyme borreliosis - a review
}

\author{
Sebastian Udziela ${ }^{1}$, Grażyna Biesiada², Magdalena Osiewicz ${ }^{3}$, Mateusz Michalak4, \\ Katarzyna Stażyk², Aleksander Garlicki², Jacek Czepiel²
}

\author{
${ }^{1}$ Independent Public Regional Hospital, Szczecin, Poland \\ ${ }^{2}$ Department of Infectious and Tropical Diseases, Jagiellonian University Medical College, \\ Krakow, Poland \\ ${ }^{3}$ Department of Integrated Dentistry, Dental Institute, Faculty of Medicine, \\ Jagiellonian University Medical College, Krakow, Poland \\ ${ }^{4}$ Students' Scientific Society Jagiellonian University Medical College, Krakow, Poland
}

Submitted: 12 November 2019; Accepted: 23 March 2020

Online publication: 17 June 2020

Arch Med Sci 2022; 18 (3): 726-731

DOI: https://doi.org/10.5114/aoms.2020.96458

Copyright $\odot 2020$ Termedia \& Banach

\begin{abstract}
Lyme borreliosis (LB) is a zoonotic disease caused by Gram-negative bacteria Borrelia burgdorferi sensu lato. The majority of reported cases of LB originate in the northern hemisphere, mostly in the US and Europe. One of the typical manifestations of LB are musculoskeletal symptoms; they may appear in any of the three LB stages. The diagnosis is based on clinical manifestations and confirmed by serological tests. One course of antibiotic therapy is sufficient for LB to dissipate in most cases, although for some patients, the symptoms subside gradually even after completion of therapy. Patients who have been demonstrated to have specific antibodies but are symptomless should not be treated. In instances where the advised treatment proved to be unsuccessful, patients should be referred to rheumatologist for additional diagnostics. The goal of this review is to update physicians on current scientific knowledge on musculoskeletal manifestations of LB.
\end{abstract}

Key words: Borrelia, diagnosis, Lyme arthritis, symptoms, treatment.

\section{Introduction}

Lyme borreliosis (LB) is a zoonotic disease caused by Gram-negative bacteria Borrelia burgdorferi sensu lato (B. burgdorferi s.l.), belonging to the Spirochaetaceae family. So far, at least 20 genospecies of spirochetes constituting the $B$. burgdorferi s.l. complex have been discovered, the preeminent pathogens being $B$. burgdorferi sensu stricto (B. burgdorferi s.s.), $B$. afzelii, and B. garinii; a few other species are considered nosogenic (B. lusitaniae, B. valaisiana, and B. spielmani) $[1,2]$. The last couple of years have been marked by a re-examination of spirochete taxonomy - the new classification and the name Borreliella have been established, but they have not been universally adopted [3, 4]. The vectors of Borrelia burgdorferi $(\mathrm{Bb})$ spirochetes are tics of the genus Ixodes. Pathogen transmission occurs during the bite of a tick consuming the host's blood [5]. A typical clinical picture of LB may include symptoms involving skin, joints, nervous system, and more rarely heart and eyes. Some infected patients are asymptomatic $[1,6]$. The majority of reported cases of LB originate in the northern hemisphere, mostly in the US and Europe [7]. Clinical presen-

\author{
Corresponding author: \\ Jacek Czepiel \\ Department of Infectious \\ and Tropical Diseases \\ Jagiellonian University \\ Medical College \\ Krakow, Poland \\ E-mail: \\ czepiel.jacek1@gmail.com
}


tation of LB includes three stages: early localised infection, early disseminated infection, and late disseminated infection [8]. This review is focused on musculoskeletal manifestations in LB, which will aid clinicians in diagnosis. Various musculoskeletal symptoms may be present in a wide array of conditions, while the presence of anti-borrelial antibodies in the general population could contribute to misdiagnosis. Lyme arthritis (LA) is one of the fundamental LB symptoms; it can be defined as the presence of arthritis with swelling and pain of one or several large joints, sometimes also pain in tendons, muscles, and/or bones in patients with serologically confirmed LB $[2,9,10]$.

\section{Pathogenesis of Lyme arthritis}

Among the genospecies of $\mathrm{Bb}, \mathrm{B}$. burgdorferi s.s. demonstrates the strongest potential to damage joints [5]. It is the most common cause of LB in the United States; infection with this pathogen occurs in Europe much less commonly [5, 11, 12]. The basis of LA pathogenesis is a strong inflammatory reaction [1]. This reaction results in a perivascular collection of lymphocytes, macrophages, as well as scattered mast cells in the interstitium, typically found on histopathology in patients with severe LA [13]. Neutrophils, on the other hand, play a part in the initial phase of inflammation, subsequently being replaced by lymphocytes and plasma cells [14]. Inflammatory response of Th1 lymphocytes and cytokines: IFN- $\gamma$ and, to a lesser extent, interleukin (IL)-4, IL-10, and IL-12 play an important role in LA [15]. There is mounting evidence that in addition to Th1 lymphocytes, Th17 cells are crucial in LA development. Synovial fluid of patients with LA contains IL-17-producing T cells as well as significantly higher IL-17 concentration than the control group $[16,17]$. As mentioned earlier, the inflammatory reaction is paramount in LA development because $\mathrm{Bb}$ spirochetes do not produce toxins. However, recent studies have shown that Bbs express protease in aggrecanase activity, possibly contributing to direct damage to joint tissues. Aggrecan is a critical component of both the cartilage structure and the function of a joint [18]. Additionally, Bbs can influence the expression of a number of matrix metalloproteinases (MMPs), and aggrecanase ADAMST-4 in chondrocytes in vitro, MMP, and ADAMST-4 values are elevated in synovial fluid of LA patients [19-22]. It has been suggested that in an inflammatory reaction this leads to progressive degradation of aggrecan by host mechanisms, advancing chondral destruction and subsequent joint damage in LA [1].

\section{Clinical picture}

Musculoskeletal symptoms may appear in any of the three stages, even early localised infection, often leading to miscategorisation of patients into the wrong stage.

In the early localised stage, during which erythema migrans (EM) is a classic symptom, some patients experience migratory muscle and joint pain. Nadelman et al., in a prospective assessment of 79 patients with culture-confirmed EM, showed the presence of myalgia and arthralgia in almost half of the cases (correspondingly, 44\% and 44\%). Those symptoms are a non-specific reaction of the immune system to the infection and cannot function as a basis for diagnosis of LA [23].

In the next, early disseminated stage, arthritis may appear from a few days to months since the point of infection. The time between tick bite and the onset of LA may vary. In the US, in patients having exhibited EM and not treated with an antibiotic, LA developed in a period of 4 days to 2 years, 6 months on average; in Europe it was from 10 days to 16 months, 3 months on average $[24,25]$. LA affects almost exclusively those not treated while EM was present or those who did not notice the EM altogether. Musculoskeletal symptoms in patients who had received prompt and appropriate treatment are extremely unlikely. Prompt treatment means starting antibiotic therapy before dissemination of infection, which usually occurs a few weeks (rarely - days) after the appearance of EM. Therefore, immediate antibiotic therapy in the first days of EM virtually eliminates the possibility of LB dissemination [1, 2]. LA constitutes approximately $28 \%$ of all forms of LB reported to CDC in the US [9]; that percentage is lower in Europe, reported as 4-8\% in epidemiological studies [26-29]. Arthritis is the main component of LA, and arthralgia may precede, accompany, or follow the inflammation, and sometimes it can be the only rheumatic manifestation of LB; however, it should be considered a less common form of LA $[5,10]$. In LA, usually there is one or few joints affected (oligoarthritis), normally no more than five, and involvement of many or most joints is atypical. Ordinarily, the joint involvement is asymmetrical and concerns mostly large joints, particularly the knee; small joints may be affected as a component of oligoarthritis, whereas an isolated involvement of small joints (e.g. of the hand) point to a cause other than LA. Apart from the knees, LA commonly affects the shoulder, elbow, wrist, and ankle joints [1, 2, 30-32]. Early research into the course of LA frequently noted involvement of the temporomandibular joint $[24,33]$. According to Heir and Fein, it was the fourth most involved joint, following the knee, shoulder, and elbow [34]. However, in the following years that involvement was reported only sporadically, more often noting symptoms of temporomandibular disorders in the masticatory muscles [35-38]. The inflamed joints become swollen and painful; however, the pain 
is not severe considering the exudate present in those joints. The skin above is warm but usually not red. Most patients report limited mobility in the affected joints, and some report fatigue or malaise, but other systemic symptoms are rare $[1,39,40]$. Typically, arthritis in the course of LB is accompanied by inflammation of the synovial membrane, and less often erosion and destruction of joint structures, which can be present in longer duration arthritis [41].

Symptom duration of at least 1 year allows diagnosis of the patient with chronic LB [8]. So far, few studies have attempted to assess long-lasting impairment of the musculoskeletal system years after treatment. Gerber et al., in a prospective study of 90 children from the US, demonstrated that a few years after antibiotic therapy none of the children exhibited joint inflammation, while $4 \%$ suffered from mild to moderate musculoskeletal impairment, hindering physical activity [40]; a slightly larger percentage (8\%) was observed in the European population $[42,43]$.

\section{Diagnostic considerations}

Diagnosis of LA must be started with a thorough history and examination of the patient. The history should contain information on: previous stays in tick-endemic regions, tick bites, length of time the tick had remained attached, and method of removal. It bears noting that approximately $27-42 \%$ of European patients do not remember the tick bite at the site of the EM, and up to $75 \%$ in the US; therefore, its absence in history does not exclude LB $[6,44-47]$. The next diagnostic step is laboratory testing, performed only in patients whose reported symptoms correspond to the clinical picture of $\operatorname{LB}[1,2]$.

Serology detects specific IgM, appearing in the blood 1-2 weeks after infection, peaking at 26 weeks, and specific lgG, appearing 2-6 weeks after infection, peaking at 4-6 months. The standard is two-tier serological testing in which the first step is a sensitive enzyme-linked immunosorbent assay (EIA). If the EIA is positive or equivocal, then separate IgM and IgG immunoblots should be performed. A positive immunoblot test with a positive or borderline EIA test confirms the presence of specific $\mathrm{Bb}$ antibodies. Because both the EIA and immunoblot may be falsely positive, it is not recommended to skip the first step (EIA) [1, 2, $8,10]$. When the symptoms are relatively recent (2-4 weeks) and serological testing is negative, it should be repeated after an additional 2 weeks $[5,30]$. High antibody titre (IgM and IgG) may persist for many years, even with successful antibiotic therapy; therefore, serology does not differentiate between active infection and past infection or exposition to $\mathrm{Bb}$ in the past $[1,40]$. With that in mind, it is not recommended to test antibody levels post-treatment, given that they have no relation to the clinical picture of the disease [2].

The next diagnostic step in LA may be employing polymerase chain reaction (PCR), detecting DNA of $\mathrm{Bb}$ spirochetes in synovial fluid and/or membrane [10]. However, it has some constraints. The PCR test result will be positive in the presence of both alive and dead spirochetes. Its sensitivity for synovial fluid approximates $60-85 \%$, meaning that a negative result does not exclude LA $[1,8,10]$. In the majority of patients pre-treatment, $\mathrm{Bb}$ DNA is usually found in the synovial fluid and the synovial membrane, whereas post-treatment with antibiotics, that result is usually negative $[1,5]$. Synovial fluid itself is also employed in the diagnostic process of LA. It would be inflammatory, characterised by an increased WBC count, typically from $46,000 / \mathrm{mm}^{3}$ to $60,000 / \mathrm{mm}^{3}$ (in rare cases reaching more than $100,000 / \mathrm{mm}^{3}$ ) and dominated by neutrophils. It is usually collected to exclude other causes of arthritis, such as gout or bacterial arthritis. It is not recommended to use synovial fluid for Borrelia cultures $[6,47,48]$. Bb culturing is possible; however, the technique is expensive, requires additional laboratory conditions, and yields results after 2-6 weeks. Culturing Bb uses a rich BSK (Barbour, Stoenner, Kelly) medium and its modifications [49]. It is worth noting that a negative culture result does not exclude LB [10].

Complete blood count usually yields normal results for WBC, platelets, haemoglobin, and haematocrit. Only at an early stage of LA, in approximately $20 \%$ of patients in Europe, a small increase in aspartate transaminase and alanine transaminase may be observed. Every stage of LA may show a slight increase in erythrocyte sedimentation rate and/or C-reactive protein [5]. Medical imaging of the joints may reveal some essential data as well. Sonography or MRI might show fluid in the affected joint and thickening of the synovial membrane. X-rays of patients with chronic LA may show destruction of the joints, such as joint space narrowing, geodes, or erosion of cartilage; however, those signs are not LA specific and their prevalence is unknown. Therefore, having found joint deterioration, it is recommended to look for causes other than LA first $[1,41]$.

Serological methods used in diagnosing LB have their limitations. Seroreactivity after successful treatment of Lyme borreliosis may persist for years $[8,10]$. Another diagnostic challenge is the possibility of false positive test results, possibly caused by cross-reactivity with other spirochaetal or bacterial antigens, as well as viral antigens, autoantigens [50-52]. Because using whole-cell lysates in ELISA is hindered by a greater possibility of cross-reactivity with other pathogenic bacteria, it is recommended that laborato- 
ries use at least a second generation ELISA test based on a recombinant antigen, which decreases the likelihood of cross-reactivity. It is paramount to use multiple antigens typical only for pathogenic Borrelia strains and characterised by high binding ability to IgG and IgM [50]. Two-tier testing has low sensitivity in the early stages of the disease, due to the time needed for an immune response of the body, while the procedure itself is time-consuming. Therefore, several methods of one-tier testing, which would combine the speed and sufficient sensitivity in the early phase of LB, are being developed. Such tests are based on highly specific recombinant antigens or synthetic peptides for antibody detection. Moreover, using new assay platforms and analysers and multiplex assays fuels hopes for improvement of LB diagnosing, as presented in detail in an excellent review by Alasel and Keusgen [50].

\section{Treatment}

When musculoskeletal manifestations are present in patients with EM, the treatment is essentially the same as EM alone. First-line drugs include the following: doxycycline, amoxicillin, or cefuroxime orally. Dosing: doxycycline 100 mg every $12 \mathrm{~h}$, amoxicillin 500 mg every $8 \mathrm{~h}$, or cefuroxime $500 \mathrm{mg}$ every $12 \mathrm{~h}$ - duration of treatment 14 days. In LA, however, that duration is longer, at 28 days. The dosing of doxycycline and cefuroxime is as given above; however, doses of amoxicillin are 500-1000 mg each 8 h. Second-line treatment in LA is IV ceftriaxone, $2 \mathrm{~g} \mathrm{ev}$ ery $24 \mathrm{~h}$ for $14-28$ days. It has been established that if the symptoms subside only partially with oral antibiotic therapy, second-line treatment should consist of another recommended oral antibiotic, while reserving parenteral antibiotic treatment for those without any substantial clinical response $[8,10]$.

Untreated arthritis usually subsides on its own; however, it can take years - there have been cases where this form of LB persisted for 7-8 years [24, 53]. There is insufficient proof of effectiveness of additional antibiotic therapy courses; moreover, the potential side effects of such antibiotics need to be considered. Long-term antibiotic therapy, its frequent repetition, as well as combined therapy with multiple antibiotics, do not offer any benefit and should not be used [10, 30, 44, 54, 55]. At the start of therapy, approximately $7-30 \%$ of patients may experience aggravation of symptoms due to the Jarisch-Herxheimer reaction. It usually develops for 12-24 $\mathrm{h}$ from the start of treatment and is caused by sudden destruction of a large number of spirochetes. It does not require cessation of treatment and usually subsides in $48 \mathrm{~h}$ [56]. Pregnant and breastfeeding women should be treated the same as the general population with the exception of doxycycline, which should not be used (as well as in children up to 8 years of age). Patients who have been demonstrated to have specific antibodies but are symptomless should not be treated [10].

\section{Post-antibiotic Lyme arthritis}

One course of therapy is sufficient for LA to dissipate in most cases, although for some patients the symptoms subside gradually even after completion of therapy; therefore, an assessment of outcome should be performed 3 months after its end. Some patients do not experience any improvement after antibiotic therapy; hence, the term 'antibiotic-refractory LA' was created, defined as persisting synovitis for $>1$ month after two four-week oral antibiotic courses or $>2$ months after completion of intravenous ceftriaxone treatment [8]. Risk factors of antibiotic-refractory LA remain not fully identified [1]. This form of persistent joint inflammation is now being called "post-antibiotic Lyme arthritis" ( $p$-a LA), not to suggest antibiotic resistance as a cause of such a disease course [10]. There are two fundamental circumstances in which antibiotic therapy fails. The first is an erroneous diagnosis of LA, meaning a not uncommon situation where an$\mathrm{ti}$-Bb antibodies are found in a patient with musculoskeletal symptoms attributable to a different condition. Exclusion of other causes to the symptoms prompts us to consider the second option: $p$-a LA. The pathogenesis of $p-a$ LA is not fully clear. The patients are characterised by a higher prevalence of HLA-DRB1*0401 and related alleles $[57,58]$. A similar mechanism exists in rheumatoid arthritis, which suggests an autoimmune component to p-a LA [1]. Another idea is spirochete persistence. It has been demonstrated that in rare cases, $\mathrm{Bb}$ genetic material may be found in muscle tissue and synovial fluid many months after antibiotic therapy - PCR cannot, however, differentiate whether the genetic material comes from viable or non-viable organisms $[59,60]$. Furthermore, as yet, no borrelial mRNA has been found in people with a positive PCR-DNA result, which suggests that DNA would come from non-viable spirochetes [61].

Therefore, it is recommended to initially, in instances where the above-mentioned treatment proves to be unsuccessful, for patients to be referred to rheumatologist for additional diagnostics and symptomatic treatment, and the search for other causes of symptoms should be considered $[2,10,62,63]$. Possible methods of treatment for patients with $p$-a LA include the following: non-steroidal anti-inflammatory agents, disease modifying anti-rheumatic drugs (DMARDS), biologic agents, intra-articular steroids, and arthroscopic synovectomy [10]. 


\section{Conflict of interest}

The authors declare no conflict of interest.

\section{References}

1. Borchers AT, Keen CL, Huntley AC, Gerschwin ME. Lyme disease: a rigorous review of diagnostic criteria and treatment. J Autoimmun 2015; 57: 82-115.

2. Biesiada G, Czepiel J, Leśniak MR, Garlicki A, Mach T. Lyme disease: review. Arch Med Sci 2012; 8: 978-82.

3. Adeolu M, Gupta RS. A phylogenomic and molecular marker based proposal for the division of the genus Borrelia into two genera: the emended genus Borrelia containing only the members of the relapsing fever Borrelia, and the genus Borreliella gen. nov. containing the members of the Lyme disease Borrelia (Borrelia burgdorferi sensu lato complex). Antonie Van Leeuwenhoek 2014; 105: 1049-72.

4. Margos G, Marosevic D, Cutler S, et al. There is inadequate evidence to support the division of the genus Borrelia. Int J Syst Evol Microbiol 2017; 67: 1081-4.

5. Stanek G, Wormser GP, Gray J, Strle F. Lyme borreliosis. Lancet 2012; 379: 461-73.

6. Thompson A, Mannix R, Bachur R. Acute pediatric monoarticular arthritis: distinguishing Lyme arthritis from other etiologies. Pediatrics 2009; 123: 959-65.

7. Hubálek Z. Epidemiology of Lyme borreliosis. Curr Probl Dermatol 2009; 37: 31-50.

8. Stanek G, Strle F. Lyme borreliosis-from tick bite to diagnosis and treatment. FEMS Microbiol Rev 2018; 42: 233-58.

9. Centers for Disease Control and Prevention. Clinical manifestations of confirmed Lyme disease cases in United States, 2001-2018. Available at: www.cdc.gov.

10. Lantos P, Rumbaugh J, Bockenstedt L, et al. Draft clinical practice guidelines by the Infectious Diseases Society of America (IDSA), American Academy of Neurology (AAN), and American College of Rheumatology (ACR): 2019 Guidelines for the Prevention, Diagnosis and Treatment of Lyme Disease. Available at: https://www.idsociety. org/practice-guideline/.

11. Clark KL, Leydet B, Hartman S. Lyme borreliosis in human patients in Florida and Georgia, USA. Int J Med Sci 2013; 10: 915-31.

12. Clark KL, Leydet BF, Threlkeld C. Geographical and genospecies distribution of Borrelia burgdorferi sensu lato DNA detected in humans in the USA. J Med Microbiol 2014; 63: 674-84

13. Johnston YE, Duray PH, Steere AC, et al. Lyme arthritis. Spirochetes found in synovial microangiopathic lesions. Am J Pathol 1985; 118: 26-34.

14. Duray $\mathrm{PH}$, Steere AC. Clinical pathologic correlations of Lyme disease by stage. Ann N Y Acad Sci 1988; 539: 65-79.

15. Yin Z, Braun J, Neure L, et al. T cell cytokine pattern in the joints of patients with Lyme arthritis and its regulation by cytokines and anticytokines. Arthritis Rheum 1997; 40: 69-79.

16. Codolo G, Bossi F, Durigutto P, et al. Orchestration of inflammation and adaptive immunity in Borrelia burgdorferi-induced arthritis by neutrophil-activating protein $A$. Arthritis Rheum 2013; 65: 1232-42.

17. Infante-Duarte C, Horton HF, Byrne MC, Kamradt T. Microbial lipopeptides induce the production of IL-17 in Th cells. J Immunol 2000; 165: 6107-15.
18. Russell TM, Johnson BJ. Lyme disease spirochaetes possess an aggrecan-binding protease with aggrecanase activity. Mol Microbiol 2013; 90: 228-40.

19. Hu LT, Eskildsen MA, Masgala C, et al. Host metalloproteinases in Lyme arthritis. Arthritis Rheum 2001; 44 1401-10.

20. Lin B, Kidder JM, Noring R, Steere AC, Klempner MS, Hu LT. Differences in synovial fluid levels of matrix metalloproteinases suggest separate mechanisms of pathogenesis in Lyme arthritis before and after antibiotic treatment. J Infect Dis 2001; 184: 174-80.

21. Behera AK, Hildebrand E, Scagliotti J, Steere AC, Hu LT. Induction of host matrix metalloproteinases by Borrelia burgdorferi differs in human and murine Lyme arthritis. Infect Immun 2005; 73: 126-34.

22. Behera AK, Hildebrand E, Szafranski J, et al. Role of aggrecanase 1 in Lyme arthritis. Arthritis Rheum 2006; 54 3319-29.

23. Nadelman RB, Nowakowski J, Forseter G, et al. The clinical spectrum of early Lyme borreliosis in patients with culture-confirmed erythema migrans. Am J Med 1996; 100: 502-8.

24. Steere AC, Schoen RT, Taylor E. The clinical evolution of Lyme arthritis. Ann Intern Med 1987; 107: 725-31.

25. Herzer P. Joint manifestations of Lyme borreliosis in Europe. Scand J Infect Dis 1991; 77: 55-63.

26. Berglund J, Eitrem R, Ornstein K, et al. An epidemiologic study of Lyme disease in southern Sweden. N Engl J Med 1995; 333: 1319-27.

27. Lesnyak O, Laikovskaya E, Kufko I, Bruinink H, Baranova N, Rijpkema S. Clinical features of Lyme borreliosis in the middle Urals and distribution of Borrelia burgdorferi sensu lato species in local Ixodes persulcatus ticks. Zentralbl Bakteriol 1998; 288: 111-9.

28. Huppertz HI, Bohme M, Standaert SM, Karch H, Plotkin SA. Incidence of Lyme borreliosis in the Würzburg region of Germany. Eur J Clin Microbiol Infect Dis 1999; 18: 697703.

29. Christova I, Komitova R. Clinical and epidemiological features of Lyme borreliosis in Bulgaria. Wien Klin Wochenschr 2004; 116: 42-6.

30. Pancewicz S, Moniuszko-Malinowska A, Garlicki A, Grygorczuk S, Czupryna P, Dunaj J. Diagnostyka i leczenie Boreliozy z Lyme. Standardy Polskiego Towarzystwa Epidemiologicznego i Lekarzy Chorób Zakaźnych. 2018. Available at: http://www.pteilchz.org.pl/wp-content/uploads/2018/11/borelioza_z_lyme_2018.pdf.

31. Pancewicz SA, Rutkowski R, Rutkowski K, Zajkowska JM, Kondrusik M. Immunopathology of Lyme arthritis. Pol Merkur Lek 2007; 23: 141-4.

32. Long KC, Cohn KA. Lyme arthritis: an update for clinical practice. Pediatr Emerg Care 2018; 34: 588-91.

33. Steere AC, Malawista SE, Snydman DR, et al. Lyme arthritis: an epidemic of oligoarticular arthritis in children and adults in three Connecticut communities. Arthritis Rheum 1977; 20: 7-17.

34. Heir GM, Fein LA. Lyme disease: considerations for dentistry. J Orofac Pain 1996; 10: 74-86.

35. Wolańska-Klimkiewicz E, Szymańska J, Bachanek T. Orofacial symptoms related to boreliosis-case report. Ann Agric Environ Med 2010; 17: 319-21.

36. te Veldhuis E, Lobbezoo F, te Veldhuis A, Visscher C, Naeije $M$, van Selms M. Residual orofacial complaints following Lyme neuroboreliosis: an unusual case of TMD. J Craniomandib Funct 2011; 3: 221-8.

37. Osiewicz M, Manfredini D, Biesiada G, et al. Differences between palpation and static/dynamic tests to diag- 
nose painful temporomandibular disorders in patients with Lyme disease. Clin Oral Investig 2019; 23: 4411-6.

38. Osiewicz M, Manfredini D, Biesiada G, et al. Prevalence of function-dependent temporomandibular joint and masticatory muscle pain, and predictors of temporomandibular disorders among patients with Lyme disease. J Clin Med 2019; 8: 929.

39. Deanehan JK, Kimia AA, Tan Tanny SP, et al. Distinguishing Lyme from septic knee monoarthritis in Lyme dis ease - endemic areas. Pediatrics 2013; 131: 695-701.

40. Gerber MA, Zemel LS, Shapiro ED. Lyme arthritis in children: clinical epidemiology and long-term outcomes. Pediatrics 1998; 102: 905-8.

41. Arvikar S, Steere A. Diagnosis and treatment of Lyme arthritis. Infect Dis Clin N Am 2015; 29: 269-80.

42. Renaud I, Cachin C, Gerster JC. Good outcomes of Lyme arthritis in 24 patients in an endemic area of Switzerland. Joint Bone Spine 2004; 71: 39-43.

43. Bentas W, Karch H, Huppertz HI. Lyme arthritis in children and adolescents: outcome 12 months after initiation of antibiotic therapy. J Rheumatol 2000; 27: 2025-30.

44. Wormser GP, Dattwyler RJ, Shapiro ED, et al. The clinical assessment, treatment, and prevention of Lyme disease, human granulocytic anaplasmosis, and babesiosis: clinical practice guidelines by the Infectious Diseases Society of America. Clin Infect Dis 2006; 43: 1089-134.

45. Strle F, Videcnik J, Zorman P, Cimperman J, Lotric-Furlan S, Maraspin V. Clinical and epidemiological findings for patients with erythema migrans: comparison of cohorts from the years 1993 and 2000. Wien Klin Wochenschr 2002; 114: 493-7.

46. Strle F, Nadelman RB, Cimperman J, et al. Comparison of culture confirmed erythema migrans caused by Borrelia burgdorferi sensu stricto in New York state and Borrelia afzelii in Slovenia. Ann Intern Med 1999; 130: 32-6.

47. Milewski MD, Cruz Al Jr, Miller CP, Peterson AT, Smith BG. Lyme arthritis in children presenting with joint effusions. J Bone Joint Surg Am 2011; 93: 252-60.

48. Dart AH, Michelson KA, Aronson PL, et al. Hip synovial fluid cell counts in children from a Lyme disease endemic area. Pediatrics 2018; 141: e20173810.

49. Wang G, lyer R, Bittker S, et al. Variations in BarbourStoenner-Kelly culture medium modulate infectivity and pathogenicity of Borrelia burgdorferi clinical isolates. Infect Immun 2004; 72: 6702-6.

50. Alasel M, Keusgen M. Promising alternatives for one-tier testing of Lyme borreliosis. Clin Chim Acta 2018; 479: 148-54

51. Tjernberg I, Kruger G, Eliasson I. C6 peptide ELISA test in the serodiagnosis of Lyme borreliosis in Sweden. Eur J Clin Microbiol Infect Dis 2007; 26: 37-42.

52. Seriburi V, Ndukwe N, Chang Z, Cox ME, Wormser GP. High frequency of false positive IgM immunoblots for Borrelia burgdorferi in clinical practice. Clin Microbiol Infect 2012; 18: 1236-40.

53. Szer IS, Taylor E, Steere AC. The long-term course of Lyme arthritis in children. N Engl J Med 1991; 325: 159-63.

54. Mygland A, Ljøstad U, Fingerle V, Rupprecht T, Schmutzhard E, Steiner I. EFNS guidelines on the diagnosis and management of European Lyme neuroborreliosis. Eur J Neurol 2010; 17: 8-16.

55. British Infection Association. The epidemiology, prevention, investigation and treatment of Lyme borreliosis in United Kingdom patients: a position statement by the British Infection Association. J Infect 2011; 62: 329-38.

56. Butler T. The Jarisch-Herxheimer reaction after antibiotic treatment of spirochetal infections: a review of recent cases and our understanding of pathogenesis. Am J Trop Med Hyg 2017; 96: 46-52.

57. Steere AC, Klitz W, Drouin EE, et al. Antibiotic-refractory Lyme arthritis is associated with HLA-DR molecules that bind a Borrelia burgdorferi peptide. J Exp Med 2006; 203: 961-71.

58. Steere AC, Dwyer E, Winchester R. Association of chronic Lyme arthritis with HLA-DR4 and HLA-DR2 alleles. N Engl J Med 1990; 323: 219-23.

59. Battafarano DF, Combs JA, Enzenauer RJ, Fitzpatrick JE. Chronic septic arthritis caused by Borrelia burgdorferi. Clin Orthop Relat Res 1993; 297: 238-41

60. Frey M, Jaulhac B, Piemont Y, et al. Detection of Borrelia burgdorferi DNA in muscle of patients with chronic myalgia related to Lyme disease. Am J Med 1998; 104: 591-4.

61. Li X, McHugh GA, Damle N, Sikand VK, Glickstein L, Steere AC. Burden and viability of Borrelia burgdorferi in skin and joints of patients with erythema migrans or Lyme arthritis. Arthritis Rheum 2011; 63: 2238-47.

62. Jedynak M, Siemiatkowski A, Milewski R, Mroczko B, Szmitkowski M. Diagnostic effectiveness of soluble triggering receptor expressed on myeloid cells-1 in sepsis, severe sepsis and septic shock. Arch Med Sci 2019; 15: 713-21.

63. Ma K, Li L, Liu C, Zhou L, Zhou X. Efficacy and safety of various anti-rheumatic treatments for patients with rheumatoid arthritis: a network meta-analysis. Arch Med Sci 2019; 15: 33-54. 